\title{
Chronische Unterbauchschmerzen und Persönlichkeit - Zusammenhänge zwischen körperlichen Beschwerden und psychischer Struktur
}

\author{
Chronic Pelvic Pain Syndrome and Personality - Association of Somatic \\ Symptoms and Psychic Structure
}

Autoren

Institut

\author{
Rebecca Albrecht, Bernd Löwe, Christian A. BrünahI ", Björn Riegel
}

Institut und Poliklinik für Psychosomatische Medizin und Psychotherapie, Psychsomatische Medizin und Psychotherapie, Universitätsklinikum Hamburg-Eppendorf \& Schön Klinik Hamburg Eilbek
Schlüsselwörter

- Chronisches Unterbauchschmerzsyndrom

- Symptome des unteren Harntraktes

- Schmerzwahrnehmung

- Psychopathologie

- Persönlichkeit

Keywords

- chronic prostatitis with chronic pelvic pain syndrome

- lower urinary tract symptoms

- pain perception

- psychopathology

personality eingereicht 15. Januar 2015 akzeptiert 14. Mai 2015

Bibliografie

DOI http://dx.doi.org/ 10.1055/s-0035-1554692

Online-Publikation: 22.7.2015

Psychother Psych Med 2015; 65: 418-425

(c) Georg Thieme Verlag KG Stuttgart · New York ISSN 0937-2032

Korrespondenzadresse Dr. med. Rebecca Albrecht Institut und Poliklinik für Psychosomatische Medizin und Psychotherapie

Universitätsklinikum Hamburg-Eppendorf Martinistraße 52 20246 Hamburg re.albrecht@uke.de

\section{Zusammenfassung}

$\nabla$

Einleitung: Das chronische Unterbauchschmerzsyndrom („Chronic Pelvic Pain Syndrome“, CPPS) ist ein Krankheitsbild mit hoher Prävalenz, ohne jedoch mit den auslösenden und aufrechterhaltenden Bedingungen hinreichend verstanden zu sein. Es ist gekennzeichnet von anhaltenden Schmerzen unterschiedlicher Intensität und Qualität im Unterbauch. Neben anderen psychosozialen Faktoren für das Verständnis des CPPS wurden zuletzt auch Zusammenhänge mit Mentalisierungs- und Bindungsstörungen diskutiert. Unsere Studie untersucht die Ausprägung von Persönlichkeitsdimensionen nach ICD-10/ DSM-IV sowie der psychischen Struktur nach OPD-2 und deren Zusammenhang mit der urologischen Symptomausprägung (erhoben mit dem NIH-Fragebogen) und der subjektiven Schmerzwahrnehmung (erhoben mit dem MPQ).

Methoden: In einer CPPS-Spezialsprechstunde wurden 109 Patientinnen und Patienten mit standardisierten Fragebögen zur Persönlichkeitsdiagnostik untersucht. Die Ergebnisse wurden mit Referenzstichproben verglichen. Zusätzlich wurden Zusammenhänge zwischen den Persönlichkeitsaspekten und der urologischen Symptomausprägung und der subjektiven Schmerzwahrnehmung erhoben. Die Auswertung basierte auf einem Datensatz, in dem fehlende Werte mittels multipler Imputation ersetzt wurden.

Ergebnisse: Im Vergleich zu den Normstichproben ließen sich nur in einzelnen Strukturdimensionen sowie im Selbstbild Auffälligkeiten finden. Darüber hinaus war die Korrelation zwischen der subjektiven Schmerzwahrnehmung und den meisten gemessenen Persönlichkeitsaspekten signifikant positiv, wohingegen die urologische Symptomausprägung nicht mit Persönlichkeitsaspekten in Zusammenhang stand.

* geteilte Letztautorenschaft

\section{Abstract \\ $\nabla$}

Background: Despite its high prevalence, little is known about the aetiology and maintenance of Chronic Pelvic Pain Syndrome (CPPS). CPPS is is considered to be a multi-causal syndrome with discomfort and pain in the pelvis. Recent literature suggests that psychosocial factors are important for understanding CPPS. For example, CPPS has been associated with deficits in mentalization and bonding experiences. Our study aims to characterize features of personality disorders according to DSM-IV and psychic structure according to OPD-2 in CPPS patients. Furthermore, we examine the association of personality aspects with urological symptoms (NIH Questionnaire) and pain perception (MPQ Questionnaire).

Materials \& Methods: Personality aspects were assessed in a total of 109 patients from our CPPS outpatient clinic using standardized questionnaires. To characterize CPPS patients, we compared the sample's scores with reference groups, mostly the general population. In addition, the associations between personality aspects and both the urologic symptoms and pain perception were assessed using correlations. Missing data were replaced using multiple imputation methods.

Results: Compared to reference values, we found 'experiencing emotions' and 'creating relationships' as specific deficits in CPPS patients. Furthermore, patients' self-image (more dominant, higher depressive mood) differs from the general population. A higher pain perception was correlated with deficits in most personality aspects we measured. However, this was not the case for the severity of urological symptoms.

Discussion \& Conclusion: Compared to the reference values, only a few personality aspects differed in CPPS patients but there was a correlational association between different personality 
Diskussion und Schlussfolgerung: Hinsichtlich der gemessenen Persönlichkeitsaspekte waren die PatientInnen mit CPPS mit den Normstichproben vergleichbar, allerdings zeigte sich ein Zusammenhang zwischen verschiedenen Persönlichkeitsaspekten und der subjektiven Schmerzwahrnehmung. Unabhängig von der urologischen Symptomausprägung war die subjektive Schmerzwahrnehmung assoziiert mit Schwierigkeiten im Umgang mit sich selbst und Anderen. Diese Erkenntnisse sollten in psychotherapeutischen Konzepten berücksichtigt werden. traits and pain perception. Despite the extend of symptoms, pain perception is associated with difficulty (emotional ability) in dealing with emotions, self-management and relationships. These personality aspects should be taken into account when planning therapy.

\section{Einleitung}

Das chronische Unterbauchschmerzsyndrom (engl. Chronic Pelvic Pain Syndrome, CPPS) ist gekennzeichnet von anhaltenden Schmerzen unterschiedlicher Intensität und Qualität und kann im Unterbauch verschiedene Lokalisationen betreffen. Es betrifft Männer und Frauen und wird in urologischen und gynäkologischen Leitlinien definiert (European Association of Urology, National Institute of Health, Gesellschaft für Psychosomatische Frauenheilkunde und Geburtshilfe). Die Symptome betreffen einzelne Organsysteme (z.B. „Chronic Prostatitis Syndrome“, „Chronic Bladder Pain Syndrome“ oder "Chronic Vulva Pain Syndrome“) oder betreffen assoziierte Strukturen wie Muskeln und Sehnen (z. B. Beckenbodenschmerzen, Schmerzen bei Harndrang). Es verursacht einen starken Leidensdruck, ohne jedoch hinsichtlich der auslösenden und aufrechterhaltenden Bedingungen hinreichend verstanden zu sein. Die Prävalenzangaben unterliegen weltweit abhängig von der untersuchten Grundgesamtheit und den genutzten Instrumenten großen Schwankungen. Bei Frauen werden Punktprävalenzen bis $25 \%[1,2]$ und bei Männern bis zu $18 \%[3,4]$ berichtet.

Die Beschwerden wie auch die vermutete Pathogenese umfassen sowohl im Unterbauch gelegene Organe und Strukturen als auch psychische Einflussfaktoren [5,6]. Dieser Komplexität wird mithilfe eines multifaktoriellen Modells Rechnung getragen, das ein Zusammenspiel aus infektiologischen, urogenitalen, neurologischen und psychologischen Faktoren beachtet [6]. Mit dem „UPOINT“-System [7] fand dieses multifaktorielle Verständnis eine konzeptionelle Umsetzung zur Klassifikation und ist in den aktuellen europäischen Leitlinien der urologischen Fachgesellschaft verankert [8], ( $\bullet$ Abb. 1).

Die Forschung hat sich bisher überwiegend auf die somatischen Faktoren bei der Entstehung, Aufrechterhaltung und Behandlung des CPPS konzentriert. In einem aktuellen systematischen

\begin{tabular}{|ll}
$\mathrm{U} \longrightarrow$ Urinary & $\begin{array}{l}\text { (Dringlichkeit und Frequenz des Harnlassens) } \\
\mathrm{P} \longrightarrow \text { Psychosocial }\end{array}$ \\
$\mathrm{I} \longrightarrow$ Organ specific & $\begin{array}{l}\text { (v.a. Depression, katastrophisierende } \\
\text { Gedanken) } \\
\text { (Druckempfindliche Prostata; nachweisbare } \\
\text { Leukozyten, Hämatospermie, schmerzhafte } \\
\text { Blasenentleerung) } \\
\text { (unterschiedliche Erreger im urogenitalen } \\
\text { Bereich) }\end{array}$ \\
$\mathrm{N} \longrightarrow$ Infection & $\begin{array}{l}\text { (z.B. neuropathische Schmerzen bei Diabetes } \\
\text { mellitus) }\end{array}$ \\
$\mathrm{T} \longrightarrow$ Tenderness & (z.B. Triggerpunkte im Beckenbodenbereich)
\end{tabular}

Abb. 1 Das UPOINT-Modell zur Klassifikation bei CPPS.
Literaturreview fanden wir Daten zu einer Vielzahl an beteiligten psychologischen Faktoren und Ko-Morbiditäten, jedoch existieren kaum qualitativ hochwertige Studien, die sich dezidiert psychosomatischen Zusammenhängen widmen [9]. Die am besten untersuchten beteiligten Faktoren sind dabei katastrophisierende Kognitionen sowie Stress. In eigenen Untersuchungen fanden wir eine deutlich erhöhte psychopathologische Belastung einhergehend mit einer stark reduzierten Lebensqualität [10] sowie einen negativen Einfluss aktuell erlebter Stressoren [11].

Entsprechend dieser Datenlage wurden bisher meist nur eine komorbide Depression, eine angstassoziierte Symptomatik sowie katastrophisierende Kognitionen zur Einschätzung der psychosozialen Domain P des UPOINT-Systems betrachtet. Persönlichkeitsfaktoren sowie Besonderheiten der psychischen Struktur der Patienten spielen in der aktuellen Debatte um beteiligte psychosoziale Einflüsse dagegen kaum eine Rolle. Eine ältere Studie postulierte bei Männern mit chronischer Prostatitis eine Persönlichkeitspathologie, v.a. vom Borderline-Typus sowie Narzissmus [12]. Neuere Studien untersuchen differenzierter Defizite in der Mentalisierungsfähigkeit sowie den Bindungserfahrungen bei Frauen mit CPPS [13]. In einer anderen Studie wiesen 63\% der Probanden eine Persönlichkeitsstörung auf und die Prävalenz der Borderline-Persönlichkeitsorganisation war nahezu ebenso hoch [14].

Insgesamt existiert jedoch wenig Wissen über den Zusammenhang zwischen der Symptomatik des CPPS und Persönlichkeitsfaktoren sowie den Aspekten der Symptombewältigung unter Berücksichtigung der dem Individuum zur Verfügung stehenden psychischen Struktur. Gemäß der OPD-2, einer Operationalisierung der psychodynamischen Diagnosestellung, umfasst die psychische Struktur lebensgeschichtlich erworbene, grundsätzliche psychische und interpersonelle Fertigkeiten und Kapazitäten, welche die Persönlichkeit konstituieren. Die psychische Struktur gemäß der OPD-2 lässt sich auf 4 Dimensionen erfassen: Kognitive Fähigkeiten (z.B. Selbstreflexion und Affektdifferenzierung), emotionale Fähigkeiten (z.B. Empathie und Affektmitteilung), Steuerungsfähigkeiten (z.B. Selbststeuerung und Regulierung des Objektbezugs) und die Fähigkeit zur Bindung [15].

Es ist das Ziel der vorliegenden Studie, die Psychopathologie des Patientenklientels auf der Ebene der Persönlichkeitsstruktur zu charakterisieren. Dies umfasst sowohl die Ausprägung der Persönlichkeitsdimensionen gemäß ICD-10/DSM-IV als auch die Merkmale der psychischen Struktur nach OPD-2 sowie des Selbstbilds der Person.

Im zweiten Schritt soll in einer explorativen Analyse der Zusammenhang zwischen der urologischen Symptomausprägung und der subjektiven Schmerzwahrnehmung mit den einzelnen Aspekten der Persönlichkeitsdimensionen und der Persönlichkeitsstruktur untersucht werden. Die Studie zielt damit auf eine 
Charakterisierung der PatientInnen mit CPPS auf der Ebene der Persönlichkeit aus einem psychodynamischen Blickwinkel heraus. Die so gewonnenen Erkenntnisse geben Aufschluss über Interventionsschwerpunkte einer spezifischen psychotherapeutischen Intervention zur Behandlung des CPPS im Rahmen einer interdisziplinären Behandlungsstrategie.

\section{Stichprobe und Methoden}

$\nabla$

Im Rahmen einer querschnittlichen Studie wurden Frauen und Männer mit chronischen Schmerzen im Unterbauch- und Beckenbereich in einer interdisziplinären Spezialsprechstunde zum chronischen Unterbauchschmerzsyndrom (CPPS) am Universitätsklinikum Hamburg-Eppendorf untersucht. Dabei durchlief jeder Patient innerhalb eines Tages einen diagnostischen Algorithmus, der bereits an anderer Stelle ausführlich beschrieben ist [10]: Nach einem psychosomatisch orientierten Erstgespräch fand eine urologische Diagnostik sowie eine physiotherapeutische Examination statt. Frauen wurden zusätzlich gynäkologisch untersucht. In einer interdisziplinären Fallkonferenz fand eine Gesamtbefundschau statt, aus der die Diagnose sowie Behandlungsempfehlungen abgeleitet wurden. Die Diagnostik eines CPPS erfolgte nach den Leitlinien der European Association of Urology [8]. Gemäß der international gängigen Einteilung der NIH-Konsensuskonferenz leiden die eingeschlossenen Patienten unter „Chronic Prostatitis/Chronic Pelvic Pain Syndrome (CP/CPPS) Kategorie III [16]. Ausschlusskriterien für die Studie waren: fehlende Einwilligungserklärung, Minderjährigkeit, unzureichende Deutsch-Kenntnisse, Substanzmittelabhängigkeit mit Ausnahme von Tabak und Schmerzmitteln, akut behandlungsbedürftige psychiatrische oder medizinische Symptomatik (z.B. Suizidalität, maligner Befund).

Ein zustimmendes Votum zum Studienprotokoll der Ethikkommission der Ärztekammer Hamburg lag vor.

Die testdiagnostische Erhebung der bestehenden CPPS -relevanten urologischen Symptomausprägung erfolgte mit dem international gängigen Forschungsinstrument „National Institutes of Health Chronic Prostatitis Symptom Index“ (NIH-CPSI) [17]. Der NIH-CPSI liegt auch in einer deutschen Übersetzung vor, die Re-Test-Reliabilität beträgt $r=0,83-0,93$. Validitätsuntersuchungen mit anderen Patientengruppen liegen vor [18]. 9 Items auf 3 Skalen (Schmerz, urologische Symptome, Lebensqualität) messen die Symptomausprägung. Für jede Skala kann ein Summenwert errechnet werden. Der Summenwert der 3 Skalen bildet den Gesamtwert. Die deutsche Evaluierung [18] berichtet einen Gesamtwert von 22,7 bei einer Stichprobe von Patienten mit CPPS. Dieser ist mit dem Gesamtwert in der vorliegenden Studie vergleichbar.

Die subjektive Schmerzwahrnehmung wurde mit der deutschen Version der Kurzform des McGill Pain Questionnaire gemessen (SF-MPQ) [19]. Mit insgesamt 15 Adjektiven wird die Ausprägung der sensorischen (11 Items) sowie der affektiven Qualität (4 Items) von neuropathischen wie nicht-neuropathischen Schmerzen gemessen. Der Gesamtwert gibt einen Überblick über das Ausmaß der Belastung durch die subjektive Schmerzwahrnehmung; Referenzdaten (Gesamtwert=14,7) liegen vor [20].

Die Persönlichkeitsaspekte sowie die psychische Struktur wurden mit 3 Selbstbeurteilungsinstrumenten gemessen. Das Persönlichkeitsstile und -störungen Inventar (PSSI) [21] erfasst mit 140 Items 14 verschiedene Persönlichkeitsdimensionen nach ICD-10 und DSM-IV, z.B. die Dimensionen ruhig vs. depressiv oder spontan vs. Borderline. Dabei bilden die Endpunkte der Dimensionen nicht zwangsläufig Gegensätze, sondern werden als 2 mögliche Ausprägungen einer Persönlichkeitsdimension verstanden. Der PSSI wurde populationsbezogen normiert; zur Auswertung liegen T-Normen vor, Cronbachs $\alpha=0,73-0,85$ je nach Skala; die Validität differenziert zwischen verschiedenen Patientengruppen.

Der Gießen-Test II (GT-II) ermöglicht eine Erhebung des Selbstbildes [22]. Die 40 bipolar dargebotenen Items des Tests sind 6 faktorenanalytisch begründeten Skalen zugeordnet, die jeweils als dimensionale Ausprägungen zu verstehen sind, z.B. Dominanz vs. Gefügigkeit. Sowohl in der therapeutischen Praxis als auch in der Forschung gilt der GT-II als arriviertes Instrument, das an einer bevölkerungsrepräsentativen Stichprobe zuletzt 2006 normiert wurde. Die Re-Test Reliabilität liegt je nach Studie zwischen 0,59 und 0,72. Auch Validitätswerte sind hinreichend belegt (u.a. mit dem Neo-FFI) [22].

Zur Erfassung der psychischen Struktur wurde der Selbstauskunftsfragebogen zur Strukturachse der OPD-2 (OPD-SF) genutzt [15]. Die 95 Items des Fragebogens sind aus dem Interview zur Strukturachse der OPD-2 abgeleitet und bilden die einzelnen strukturellen Dimensionen ab, z.B. Selbstreflexion oder Affektdifferenzierung. Jede Dimension lässt sich gesondert auswerten, wobei der Summenwert den Gesamtwert für die psychische Struktur angibt. Je höher der Wert, desto niedriger integriert ist die psychische Struktur. Dies bedeutet, dass mehr Defizite im Umgang mit sich und anderen bestehen. Die interne Konsistenz wird mit einem Reliabilitätswert Cronbachs $\alpha=0,65-0,82$ je nach Subskala angegeben. Der OPD-SF wurde an einer nichtklinischen, einer ambulant-psychotherapeutischen und einer stationär-psychotherapeutischen Stichprobe hinsichtlich der Validität geprüft. Der Gesamtwert des OPD-SF konnte zwischen den einzelnen Gruppen differenzieren. Die Ergebnisse sind zufriedenstellend. Zudem konnte eine mittlere Übereinstimmung mit den Resultaten eines OPD-2 Interviews gefunden werden [15]. Die Auswertung der Ergebnisse fand mit SPSS 20.0 statt. Fehlende Werte wurden mittels SPSS-interner Multipler Imputation ersetzt, indem 200 Datensätze erzeugt und zu einem kombinierten Wert aggregiert werden. Alle ausgewerteten Variablen wurden auf Itemebene imputiert, während vollständig vorliegende Variablen (z. B. Alter, Geschlecht) als Indikatorvariablen einbezogen wurden.

Um die Sensitivität der Ergebnisse zu untersuchen, wurden die auf der Basis der Multiplen Imputation gewonnenen Daten mit den Originaldaten auf bedeutsame Abweichungen hin verglichen. Auf der Grundlage dieses imputierten Datensatzes wurden im ersten Schritt die normierten Skalenwerte des PSSI (geschlechtsspezifische T-Norm) errechnet. Zudem wurden die Extremwerte (T-Wert $>2 S D$ ) in den einzelnen Persönlichkeitsdimensionen deskriptiv dargestellt. Die Skalenwerte des GT-II und des OPD-SF wurden mittels eines T-Tests für eine Stichprobe mit der Normstichprobe (GT-II) bzw. mit der nicht-klinischen Vergleichsgruppe (OPD-SF) verglichen. In einem zweiten Schritt wurden Korrelationen zwischen den 3 Persönlichkeitsinstrumenten und der urologischen Symptomausprägung (NIH-CPSI) sowie der subjektiven Schmerzwahrnehmung (SF-MPQ) berechnet. Das Signifikanzniveau wurde mit $\mathrm{p}<0,05$ festgelegt. Gemäß der Einteilung von Cohen [23] wurden Korrelationen $r<0,30$ als schwach angesehen; bis $r=0,50$ wurde als mittlerer Zusammenhang und Korrelationen $r>0,50$ als starker Zusammenhang bewertet. 


\section{Ergebnisse}

Im Zeitraum von 10/2012 bis 11/2014 wurden 126 Patientinnen und Patienten in der interdisziplinären Spezialsprechstunde CPPS am Universitätsklinikum Hamburg-Eppendorf vorstellig, von denen 109 in die Studie aufgenommen wurden (57\% Frauen). Ausschlussgründe waren das Fehlen oder der Rückzug der Einwilligungserklärung $(n=4)$, keine Diagnose eines CPPS $(n=9)$ und mangelnde Deutschkenntnisse $(n=4)$.

Die soziodemografische und krankheitsbezogene Charakterisierung der Stichprobe ist in 0 Tab. 1 dargestellt. Die Alterspanne der Betroffenen ist groß (19-84 Jahre), wobei 20\% der Studienteilnehmer 30 Jahre oder jünger sind. Die Patienten geben durchschnittlich eine hohe Schmerzbelastung an (VAS: 55; SD =22) und lassen ein weitgehend chronifiziertes Beschwerdebild erkennen (Symptomdauer: 6,5 Jahre; $\mathrm{SD}=7,9$ ).

Die Auswertung erfolgte auf der Basis eines imputierten Datensatzes. Die Sensitivitätsanalyse zeigte keine bedeutsamen Abweichungen von den Originaldaten in der Tendenz der Ergebnisse.

\section{Psychometrische Charakterisierung der Stichprobe im Vergleich zu Normdaten \\ $\nabla$}

Im Mittelwert zeigen sich bei unserer Stichprobe keine erhöhten Werte in der Ausprägung der Persönlichkeitsdimensionen nach ICD-10/DSM-IV (PSSI). Die Durchschnittswerte aller Skalen liegen im Normbereich ( $T=50 \pm 1 S D)$.

Bei der Betrachtung der einzelnen T-Werte in der normierten T-Wert-Skala zeigten 24,8\% der Befragten Extremwerte im hohen Bereich $(\mathrm{T}>70)$ in mindestens einer Persönlichkeitsdimension. Am häufigsten treten Extremwerte in den Dimensionen zwanghaft $(n=11 ; 10,1 \%)$, selbstunsicher und selbstlos (je $n=5 ; 4,6 \%$ ) sowie histrionisch und depressiv $(n=4 ; 3,7 \%)$ auf.

Das Selbstbild der CPPS-Stichprobe im GT-II unterscheidet sich von der Normstichprobe in den Dimensionen Dominanz $(\mathrm{T}=-2,80 ; \mathrm{p}=0,007)$ und Grundstimmung $(\mathrm{T}=6,52 ; \mathrm{p}<0,001)$. Damit beschreiben sich die PatientInnen als durchschnittlich häufiger in Auseinandersetzungen verstrickt, dominierend und ungeduldig (Skala Dominanz). Der innere Konfliktdruck wird eher impulsiv am Gegenüber abreagiert [22]. Die Grundstimmung ist dagegen depressiver gefärbt. Dies geht mit einer Tendenz zur Suche nach Abhängigkeitsbeziehungen einher [22]. Hinsichtlich der psychischen Struktur (OPD-SF) weist die CPPSStichprobe im Vergleich zur gesunden Vergleichsstichprobe signifikant höhere Werte in den Skalen Emotionale Kommunikation nach innen $(T=4,62 ; \mathrm{p}<0,001)$ und Bindung außen $(T=2,09$; $\mathrm{p}=0,041)$ auf, jedoch unterscheidet sich der Gesamtwert des OPD-SF nicht von der nicht-klinischen Vergleichsstichprobe $(\mathrm{T}=1,16 \mathrm{p}=0,248)$ ( $\bullet$ Abb. 2). In den auffälligen Dimensionen deuten höhere Skalenwerte im OPD-SF auf größere Defizite der abgefragten Dimension hin.

\section{Zusammenhänge zwischen den Persönlichkeits- maßen und der urologischen Symptomausprägung $\nabla$}

Es ergaben sich nur schwache Korrelationen zwischen spezifischen Persönlichkeitsdimensionen (PSSI) und einer hohen urologischen Symptomausprägung (NIH-CPSI) ( $\bullet$ Tab. 2). Die Skalen des GT-II hängen ebenfalls nur schwach mit der Symptomausprägung zusammen ( $\bullet$ Tab. 3). Zudem bestehen schwache positive
Korrelationen zwischen der urologischen Symptomausprägung und den Strukturdimensionen des OPD-SF ( $\bullet$ Tab. 3).

\section{Zusammenhänge zwischen den Persönlichkeits- maßen und der subjektiven Schmerzwahrnehmung $\nabla$}

Eine stärker ausgeprägte subjektive Schmerzwahrnehmung (SF-MPQ) ist in einem mittleren Ausmaß korreliert mit einer hohen Ausprägung in den Skalen zu einzelnen Persönlichkeitstypen der Cluster B (Antisozial, histrionisch, Borderline, narzisstisch) und Cluster C (abhängig, negativistisch, depressiv) des PSSI ( $\bullet$ Tab. 2). Insbesondere der narzisstische und der emotionalinstabile Persönlichkeitsstil weisen einen mittleren bzw. starken Zusammenhang mit einer hohen Belastung hinsichtlich der subjektiven Schmerzwahrnehmung auf.

Die Ergebnisse zum Selbstbild der PatientInnen (GT-II) zeigten einen schwachen positiven Zusammenhang zur subjektiven Schmerzwahrnehmung ( $\bullet$ Tab. 3).

Mit 2 Ausnahmen weisen alle Strukturdimensionen mittlere positive Zusammenhänge mit dem Ausmaß der subjektiven Schmerzwahrnehmung. Auch die Korrelation des Gesamtwertes des OPD-SF mit der subjektiven Schmerzwahrnehmung ist in einem mittleren Bereich.

\section{Diskussion \\ $\nabla$}

Die PatientInnen gaben insgesamt eine hohe Schmerzbelastung mit Beschwerden im Urogenitaltrakt (Dysurie, Pollakisurie, Defäkationsprobleme, Dyspareunie, Schmerzen bei der Ejakula-

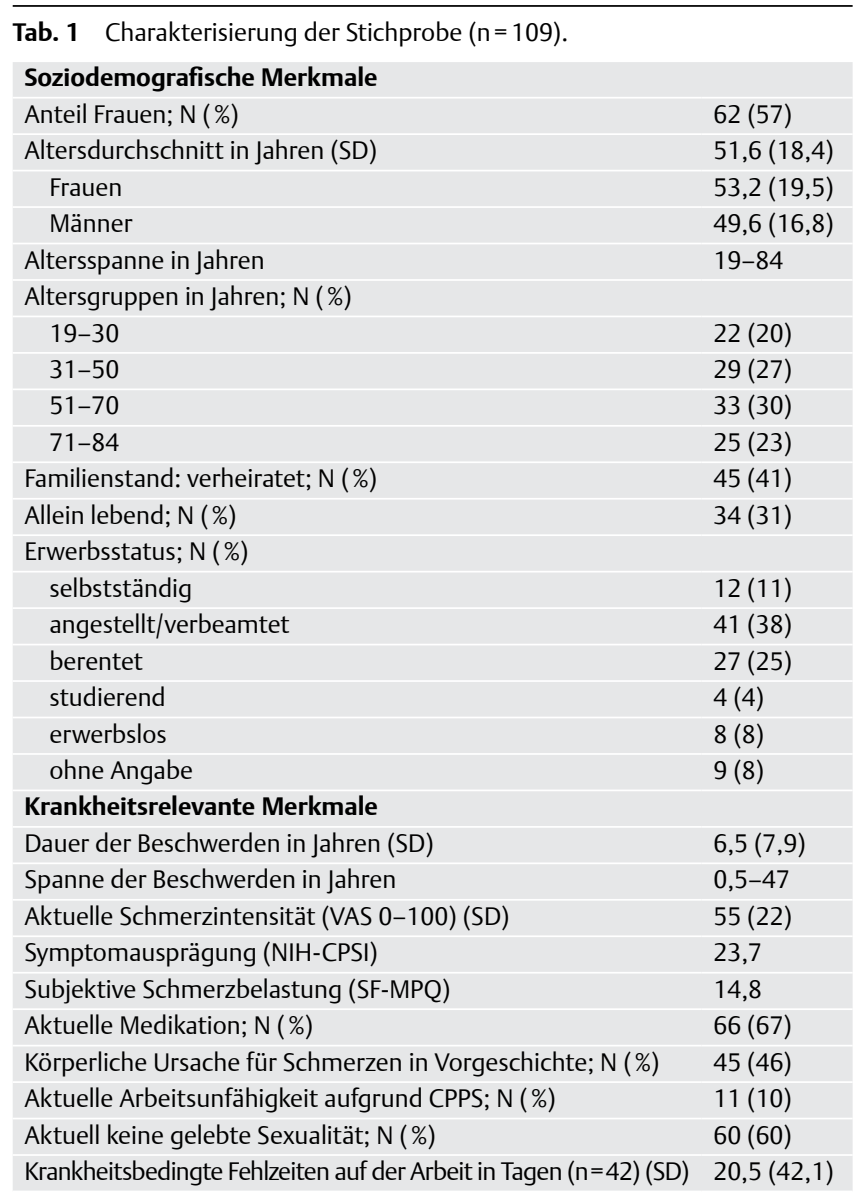


tion) an. Dabei waren die Werte in den Fragebögen zur urologischen Symptomausprägung und zur subjektiven Schmerzbelastung vergleichbar mit den Werten, die in anderen Studien berichtet wurden $[18,20]$. Anhand der erhobenen Daten konnte eine Charakterisierung der Persönlichkeitsdimensionen und -struktur dieser Stichprobe vorgenommen werden.

Dabei zeigten sich im Vergleich zu Normstichproben keine generellen Abweichungen in den Persönlichkeitsdimensionen nach ICD-10/DSM-IV. In einzelnen Strukturanteilen der OPD-2 wurden Einschränkungen in der psychischen Struktur gemäß der OPD-2 erkennbar. Zudem beschrieben sich die PatientInnen im Vergleich zur Referenzstichprobe weniger anpassungsbereit und mit einer ausgeprägten depressiven Grundstimmung im GießenTest II.

Das zweite Ergebnis besteht in einem signifikanten Zusammenhang zwischen einer hohen subjektiven Schmerzwahrnehmung und höheren Abweichungen in den Persönlichkeitsdimensionen sowie größeren Einschränkungen in den verschiedenen Aspekten der psychischen Struktur. Dieser Zusammenhang mit den
Persönlichkeitsmaßen zeigt sich dahingegen nicht hinsichtlich der urologischen Symptomausprägung.

Die größeren Einschränkungen in den verschiedenen Aspekten der psychischen Struktur unserer Stichprobe betrafen die Affektwahrnehmung, Affekttoleranz und Affektdifferenzierung (OPDSF: „emotionale Kommunikation nach innen“) und die Bindungsfähigkeit, die Fähigkeit Bindungen zu lösen und Hilfe anzunehmen (OPD-SF: „Bindung außen“). Diese Ergebnisse sind in Übereinstimmung mit den von einer anderen Arbeitsgruppe gefundenen defizitären Mentalisierungsfähigkeiten und Bindungsstörungen bei Patientinnen mit chronischen Unterbauchschmerzen [13]. Dies könnte hindeuten auf eine Assoziation von erhöhten Schwierigkeiten in der Beziehungsgestaltung mit einer ausgeprägten subjektiven Schmerzwahrnehmung. Die daraus resultierenden interpersonellen Schwierigkeiten finden sich auch im Gießen-Test in der Skala Dominanz bzw. mangelnde Anpassungsbereitschaft wieder. Aus psychodynamischer Sicht können diese Daten ein Hinweis sein, dass dem Symptom auch eine beziehungsregulierende Funktion zukommt.

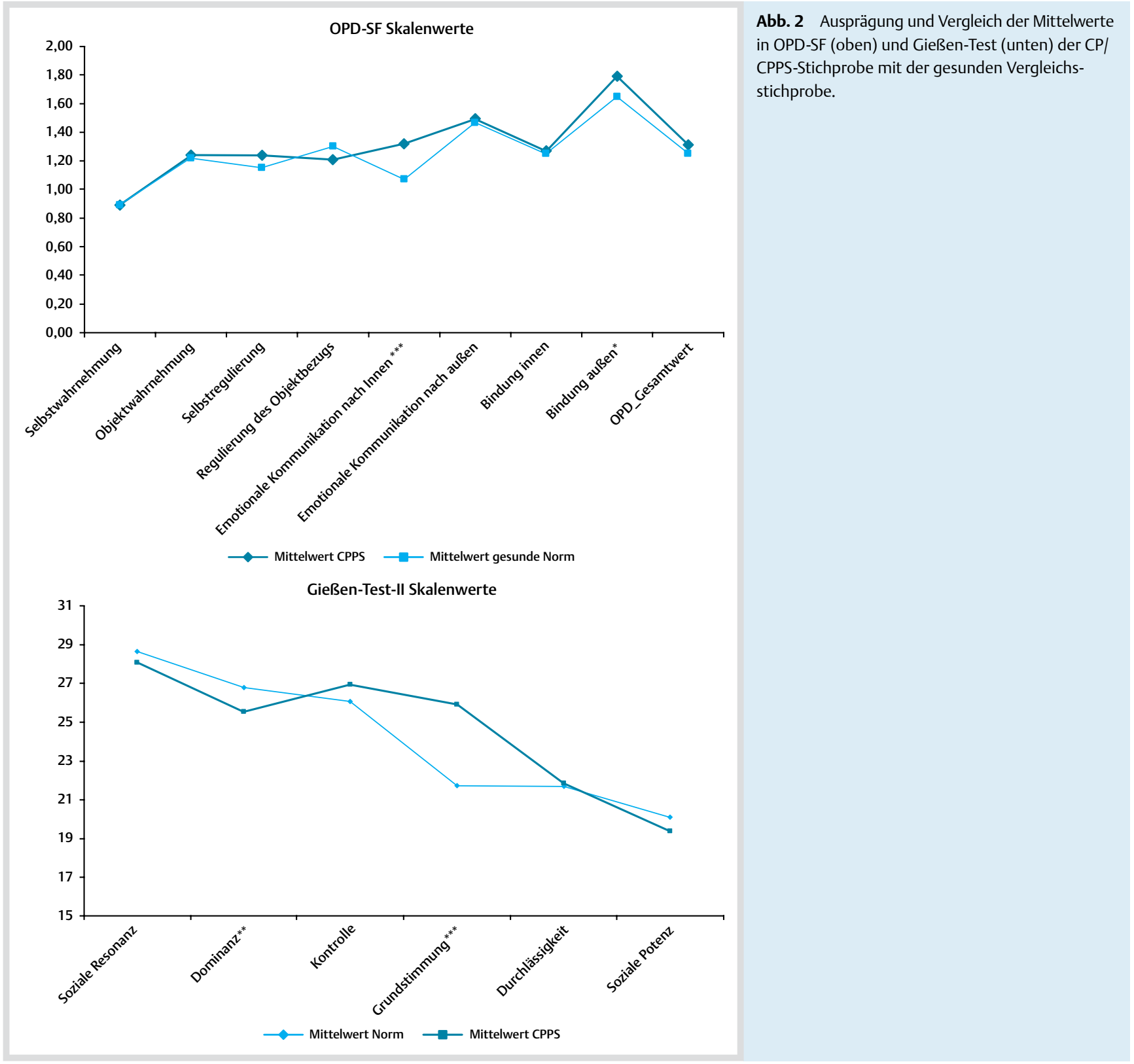



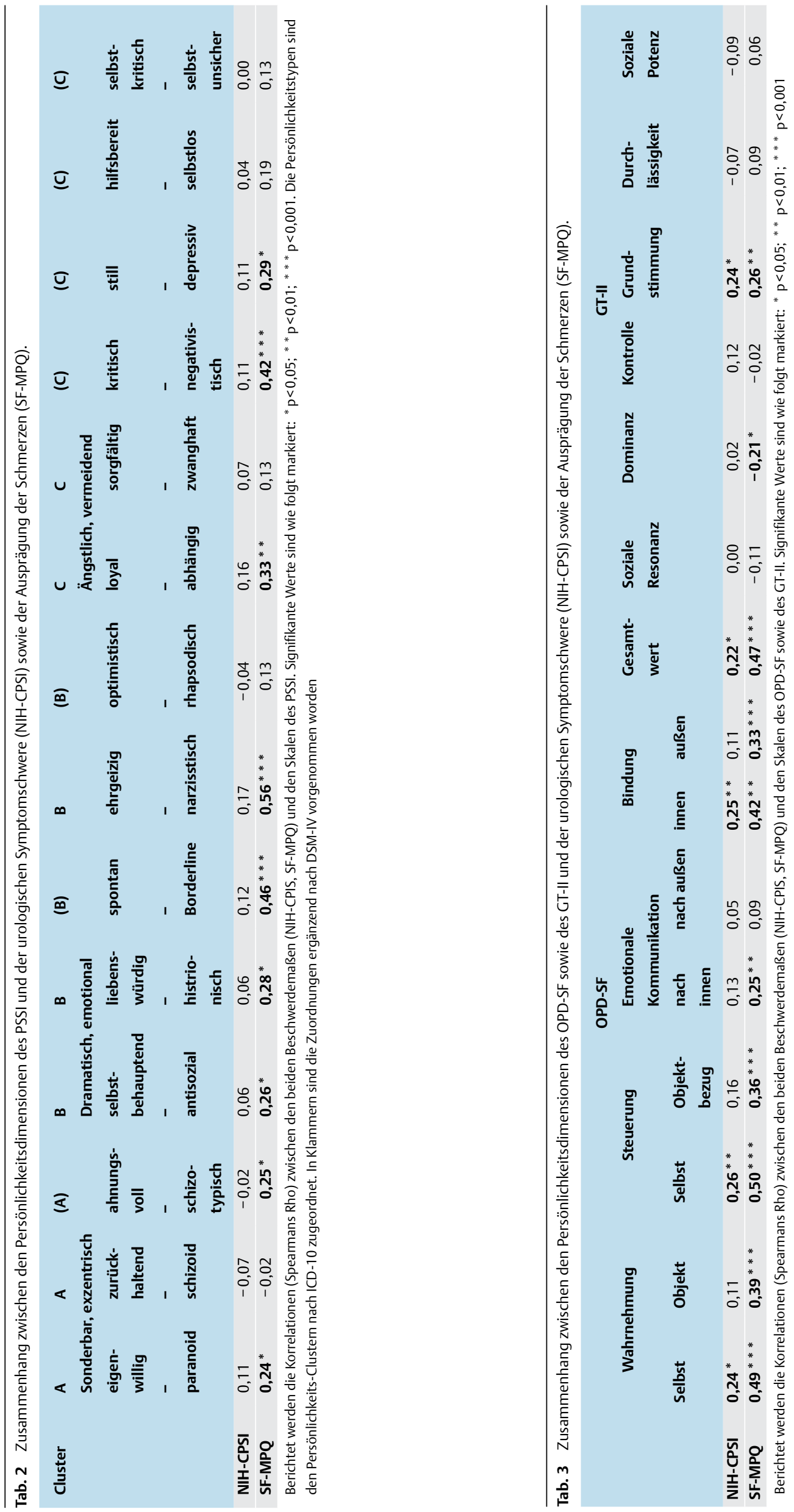
Diese Verschiebung des Fokus weg von seelischen Prozessen hin zu körperlichen Symptomen kann als Hinweis auf eine Somatisierungstendenz interpretiert werden, was wiederum die Frage aufwirft, inwiefern CPPS als somatoforme Störung begriffen werden kann. In der vorliegenden Studie sind die Kausalität sowie ätiologische Fragen aufgrund des querschnittlichen Designs nicht zu klären, sodass auch auffällige Persönlichkeitszüge als Folge der Schmerzen denkbar sind. Der Frage nach einer Konzeption des CPPS als somatoforme Störung gehen auch andere Arbeitsgruppen nach, die „Chronic Pelvic Pain“ und „Bladder Pain“ im Hinblick auf somatoforme und funktionelle Aspekte untersucht und eine hohe Übereinstimmung der Krankheitsbilder gefunden haben $[24,25]$. Es ist daher für zukünftige Forschung wichtig, Patientengruppen zu identifizieren, die weniger ein organisches Korrelat als mehr eine Einschränkung in der Persönlichkeitsstruktur haben. Für diese Hypothese spricht auch der hohe Prozentsatz (>50\%) an Patienten in unserer Stichprobe, die keine Organpathologie im Vorfeld der Schmerzen berichtet haben. Die Debatte um die Differenzialdiagnose einer somatoformen Störung wird mit unserer Arbeit damit um neue Aspekte angereichert.

Unsere Untersuchung des Zusammenhangs zwischen der subjektiven Schmerzwahrnehmung und der psychischen Struktur zeigt, dass Patienten mit hohen Werten hinsichtlich Schmerzintensität, Schmerzerfahrung und Schmerzqualität (McGill Pain Questionaire), auch eine stärkere Ausprägung in allen Persönlichkeitsclustern nach ICD10/DSM-IV aufwiesen. Das vermehrte Auftreten von Extremwerten in einzelnen Persönlichkeitsdimensionen in unserer Stichprobe wirft die Frage auf, wie der Zusammenhang zwischen Persönlichkeitspathologien und der Entstehung und Aufrechterhaltung von CPPS ist. Für ein besseres Verständnis des Krankheitsbildes sollten zukünftig jene Subgruppen von PatientInnen mit CPPS mit einer Persönlichkeitsstörung differenziert betrachtet werden, da die Interpretation möglicher Zusammenhänge aufgrund unseres Querschnittdesigns begrenzt ist. Verglichen mit den Prävalenzdaten für das Vorliegen irgendeiner Persönlichkeitsstörung in der Allgemeinbevölkerung von ca. 9\% in einer US-amerikanischen Studie [26] erscheinen die von uns berichteten Zahlen von 25\% der Probanden mit Extremwerten in den Persönlichkeitsdimensionen bemerkenswert hoch, auch wenn ein direkter Vergleich des PSSI und der Einteilung von Persönlichkeitsstörungen nur Hinweise geben kann. In älteren Studien wurden Annahmen über spezifische Persönlichkeitspathologien bei Chronischer Prostatitis untersucht [12], jedoch legen unsere Daten nahe, dass diese Fragestellung auch in Zukunft mehr Beachtung finden sollte.

Eine erhöhte subjektive Schmerzwahrnehmung stand ferner mit den psychischen Strukturmerkmalen der OPD-2 „Selbstwahrnehmung“, „Selbststeuerung“ und „Bindung“ im Zusammenhang. Dies zeigte sich dagegen hinsichtlich der urologischen Symptomausprägung (NIH-CPSI) nicht. Das könnte darauf hindeuten, dass Defizite in der psychischen Struktur (wie Selbstwahrnehmung und -steuerung; Steuerung von Emotionen und Bedürfnissen) auch die Bewältigung von Schmerzen und Beschwerden des Urogenitaltrakts erschweren - auch unabhängig von der urologischen Symptomausprägung der Beschwerden. Eine andere Forschergruppe berichtete einen ähnlichen Befund bei der Untersuchung des Einflusses von Traumatisierungen auf die Schmerzerfahrung: Die erlebten Traumatisierungen in der Kindheit und im Erwachsenenalter haben bei den untersuchten Frauen nicht im Zusammenhang mit der Schmerzintensität gestanden, wohl aber mit dem Ausmaß der schmerzassoziierten Ein- schränkungen [27]. Es ist vorstellbar, dass der Einfluss von traumatischen Erfahrungen einen moderierenden Einfluss auf den Zusammenhang zwischen Defiziten in der psychischen Struktur und dem Ausmaß der wahrgenommenen Schmerzen hat.

Neben diesen Einschränkungen im Umgang mit sich selbst zeigten sich auch Zusammenhänge zwischen der subjektiven Schmerzwahrnehmung und Einschränkungen der psychischen Struktur im Umgang mit anderen (wie Objektwahrnehmung; Regulierung des Objektbezugs). Dies könnte hindeuten auf eine Assoziation von erhöhten Schwierigkeiten in der Beziehungsgestaltung mit einer ausgeprägten subjektiven Schmerzwahrnehmung. Die daraus resultierenden interpersonellen Schwierigkeiten finden sich auch im Gießen-Test in der Skala Dominanz bzw. mangelnde Anpassungsbereitschaft wieder. Aus psychodynamischer Sicht können diese Daten ein Hinweis sein, dass dem Symptom auch eine beziehungsregulierende Funktion zukommt. Sowohl im Umgang mit sich selbst als auch im Umgang mit anderen wird über die Symptomatik eine nicht anderweitig regulierbare Affektspannung verarbeitet. Es ist anzunehmen, dass die mangelnde Anpassungsbereitschaft auch die bei chronischen Beschwerden erforderlichen Anpassungsleistungen erschwert und der depressiven Grundstimmung Vorschub leistet, die im Gießen-Test abgebildet war und sich auch in einer früheren Erhebung der Pilotphase unserer Sprechstunde in einer erhöhten psychopathologischen Belastung niederschlug [10].

Unseres Wissens handelt es sich um die erstmalige Untersuchung von Patienten mit CPPS im Hinblick auf psychische Strukturmerkmale der OPD. Die klinisch oft angenommene Schwierigkeit im Bereich der Affektwahrnehmung („Alexithymiemodell“) von Patienten mit chronischen/somatoformen Schmerzstörungen und die besondere Ausprägung von Persönlichkeitsmerkmalen („Schmerzpersönlichkeit“) konnten in unserer Stichprobe systematisch und objektiv bei PatientInnen mit CPPS untersucht werden. Die Stärken der Studie bestehen in einer intensiven interdisziplinären Diagnostik sowie der systematischen Erhebung von Persönlichkeitsaspekten mit standardisierten Messinstrumenten.

Abschließend sind einige Limitationen zu nennen, vor deren Hintergrund unsere Ergebnisse verstanden werden müssen.

Die Daten basieren auf einer einmaligen, querschnittlichen Befragung von Patienten mit CPPS ausschließlich mit Hilfe von Selbstauskunftsinstrumenten. Eine Validierung über ein strukturiertes Interview (z.B. OPD-2) fand nicht statt. Es ist zudem in diesem Forschungsdesign nicht möglich, kausale Schlussfolgerungen zu ziehen. Wir konnten lediglich Zusammenhänge aufzeigen, deren Verursachung in weiteren Studien mit einem längsschnittlichen Design sowie im Vergleich mit anderen Schmerzerkrankungen geklärt werden muss. So ist es durchaus möglich, dass sich Betroffene mit einer chronischen Schmerzsymptomatik aufgrund ihres hohen und regressiv wirkenden Leidensdrucks eingeschränkter in der psychischen Struktur einschätzen. Das querschnittliche Studiendesign lässt außerdem nur Aussagen über den momentanen Zustand zu, während keine Daten über die frühere psychische Entwicklung vorliegen. So bleibt unklar, ob die Auffälligkeiten als prämorbide psychische Strukturen oder eher als Begleit- oder Folgesymptomatik zu verstehen sind.

Die Stichprobe setzt sich aus Patienten zusammen, die in einer universitären Spezialsprechstunde vorstellig wurde und unterliegt damit einer Selektion. Es ist nicht auszuschließen, dass es sich um überwiegend schwere oder chronifizierte Krankheitsverläufe handelt, die nicht repräsentativ für das Gesamtklientel 
der Patienten mit CPPS sind. Auch der hohe Anteil von Patienten mit Auffälligkeiten in der Persönlichkeitsstruktur bzw. mit Persönlichkeitsakzentuierung könnte einer Selektivität der Stichprobe geschuldet sein.

\section{Förderung}

Die interdisziplinäre Forschungsplattform „Chronic Pelvic Pain Syndrome (CPPS)“ wird von der PRANA-Stiftung im Stifterverband der deutschen Wissenschaft gefördert. Die PRANAStiftung war weder in die Datenanalyse noch im Erstellen des Manuskripts oder in die Entscheidung zum Einreichen in dieser Zeitschrift involviert.

Des Weiteren wurde die vorliegende Arbeit durch den Marianne Ringler Forschungsförderungspreis 2013 gefördert. Eine Einflussnahme auf die vorliegende Arbeit erfolgte nicht.

\section{Fazit für die Praxis}

Die Ergebnisse unserer Studie geben Hinweise auf die Wichtigkeit der Beachtung von Persönlichkeitsdimensionen und psychischer Struktur bei dem Krankheitsbild CPPS. Insgesamt zeigte sich unsere Stichprobe hinsichtlich der gemessenen Persönlichkeitsaspekte nicht auffällig gegenüber den jeweiligen Normstichproben, jedoch offenbart sich ein Zusammenhang zwischen den Möglichkeiten der Wahrnehmung und Regulation von Affekten sowie der Steuerungsfähigkeit in Beziehungen und der subjektiven Schmerzwahrnehmung. Diese Ergebnisse können eine Erweiterung der Betrachtungsweise der P-Domain des UPOINT-Systems mit Hinblick auf die psychodynamischen bzw. persönlichkeitsbezogenen Aspekte anregen, müssen aber weiter empirisch untermauert werden. Insbesondere Subgruppen sind noch zu identifizieren und Risikofaktoren zu beschreiben, die dann für die Entwicklung von Indikationskriterien für eine spezifische Therapie genutzt werden können.

Die Daten bestätigen die Annahme, dass Patienten mit CPPS von einer Psychotherapie profitieren können, insbesondere im Hinblick auf die subjektive Schmerzwahrnehmung.

Interessenkonflikt: Die Autoren geben an, dass kein Interessenkonflikt besteht.

\section{Literatur}

1 Grace VM, Zondervan KT. Chronic pelvic pain in New Zealand: prevalence, pain severity, diagnoses and use of the health services. Aust $\mathrm{N}$ Z J Public Health 2004; 28: 369-375

2 Zondervan KT, Yudkin PL, Vessey MP et al. The community prevalence of chronic pelvic pain in women and associated illness behaviour. $\mathrm{Br}$ J Gen Pr 2001; 51: 541-547

3 Marszalek M, Wehrberger C, Temml $C$ et al. Chronic Pelvic Pain and Lower Urinary Tract Symptoms in Both Sexes: Analysis of 2749 Participants of an Urban Health Screening Project. Eur Urol 2009; 55: 499-508
4 Pitts M, Ferris J, Smith A et al. Prevalence and correlates of three types of pelvic pain in a nationally representative sample of Australian men. J Sex Med 2008; 5: 1223-1229

5 Baranowski AP. Chronic pelvic pain. Best Pract Res Clin Gastroenterol 2009; 23: 593-610 doi:10.1016/j.bpg.2009.04.013

6 Pontari MA, Ruggieri MR. Mechanisms in Prostatitis/Chronic Pelvic Pain Syndrome. J Urol 2008; 179: S61-S67

7 Samplaski MK, Li J, Shoskes DA. Clustering of UPOINT domains and subdomains in men with chronic prostatitis/chronic pelvic pain syndrome and contribution to symptom severity. J Urol 2012; 188: 1788-1793

8 European Association of Urology. Guidelines on Chronic Pelvic Pain. 2015; Im Internet: http://uroweb.org/guideline/chronic-pelvic-pain/ Stand: 21.05.2015

9 Riegel B, Bruenahl CA, Ahyai S et al. Assessing psychological factors, social aspects and psychiatric co-morbidity associated with Chronic Prostatitis/Chronic Pelvic Pain Syndrome (CP/CPPS) in men - A systematic review. J Psychosom Res 2014; 77: 333-350

10 Brünahl CA, Riegel B, HöinkJ et al. Psychosomatische Aspekte des chronischen Unterbauchschmerzsyndroms. Schmerz 2014; 28: 311-318

11 Riegel B, Albrecht R, Ketels $G$ et al. Symptomschwere und Belastungsfaktoren bei Patienten mit einem chronischen Unterbauchschmerzsyndrom - Implikationen für einen interdisziplinären und multimodalen Therapieansatz. Entspannungsverfahren 2014; 31: 40-57

12 Keltikangas-Järvinen L, Ruokolainen J, Lehtonen T. Personality Pathology Underlying Chronic Prostatitis. Psychother Psychosom 1982; 37: 87-95

13 Leithner-Dziubas K, Blüml V, Naderer A et al. Mentalisierungsfähigkeit und Bindung bei Patientinnen mit chronischen Unterbauchschmerzen: eine Pilotstudie. Z Für Psychosom Med Psychother 2010; 56: 179-190

14 Fischer-Kern M, Mikuttaw C, Kapusta ND et al. Psychische Struktur bei chronischen Schmerzpatienten. Z Für Psychosom Med Psychother 2010; 56: 34-46

15 Ehrenthal J, Dinger $U$, Horsch $L$ et al. Der OPD-Strukturfragebogen (OPD-SF): Erste Ergebnisse zu Reliabilität und Validität. Psychother Psychosom Med Psychol 2012; 62: 25-32

16 Krieger JN, Leroy Nyberg J, Nickel JC. NIH Consensus Definition and Classification of Prostatitis. JAMA 1999; 282: 236-237

17 Litwin MS, McNaughton-Collins M, Fowler FJ Jr et al. The National Institutes of Health chronic prostatitis symptom index: development and validation of a new outcome measure. Chronic Prostatitis Collaborative Research Network. J Urol 1999; 162: 369-375

18 Schneider H, Brähler E, Ludwig $M$ et al. Two-year experience with the german-translated version of the NIH-CPSI in patients with $\mathrm{CP} / \mathrm{CPPS}$. Urology 2004; 63: 1027-1030

19 Radbruch L, Loick G, Kiencke P et al. Validation of the German Version of the Brief Pain Inventory. J Pain Symptom Manage 1999; 18: 180-187

20 Sawyer J, Haslam L, Robinson S et al. Pain Prevalence Study in a Large Canadian Teaching Hospital. Pain Manag Nurs 2008; 9: 104-112

21 Kuhl J, Kazen M. Persönlichkeits-Stil- und Störungs-Inventar (PSSI). Göttingen: Hogrefe; 1997

22 Beckmann D, Brähler H, Richter HE. Gießen-Test - II. Göttingen: Hans Huber; 2012

23 Cohen J. Statistical power analysis for the behavioral sciences. vol. 2nd:Hillsdale, NJ: Lawrence Earlbaum Associates; 1988

24 Ehlert $U$, Heim C, Hellhammer DH. Chronic pelvic pain as a somatoform disorder. Psychother Psychosom 1999; 68: 87-94

25 Warren JW. Bladder pain syndrome/interstitial cystitis as a functional somatic syndrome. J Psychosom Res 2014; 77: 510-515

26 Lenzenweger MF, Lane MC, Loranger AW et al. DSM-IV personality disorders in the National Comorbidity Survey Replication. Biol Psychiatry 2007; 62: 553-564

27 As-Sanie S, Clevenger LA, Geisser ME et al. History of abuse and its relationship to pain experience and depression in women with chronic pelvic pain. Am J Obstet Gynecol 2014; 210: 317.e1-317.e8 